\title{
Ultrasound Evaluation of Focal Hepatic Lesions
}

\author{
Dr. Hiral Hapani ${ }^{1}$, Dr. Jagruti Kalola ${ }^{2}$, Dr. Anjana Trivedi ${ }^{3}$, \\ Dr. Anirudh Chawla \\ (P.D.U. Govt. Medical College and Civil Hospital, Rajkot, Gujarat, India)
}

\begin{abstract}
Objective: The aim of this study was to evaluate the role of ultrasound in evaluation of focal liver lesions, to study the imaging spectrum of focal hepatic lesions, to study the relative prevalence of different focal hepatic lesions, and to correlate the ultrasound findings with FNAC and/or CT scan.

Methods: The study was prospective and was carried out between September 2014 and November 2014 at the Department of Radiology, P.D.U. Govt. Medical College and Civil Hospital, Rajkot. Abdominal ultrasound and ultrasound guided-fine needle aspiration cytology and/or CT scan of 50 patients with clinical diagnosis of hepatic focal lesions or incidentally discovered focal hepatic lesions was performed.

Results: Out of 50 patients diagnosed by ultrasound, the most common focal liver lesions seen in our study were- liver abscess, hemangiomas, cysts, metastasis, primary liver tumors, contusions/lacerations and hydatid cysts. Correlating with FNAC and/or CT scan, ultrasound had an average specificity of 93.6\%, with 100\% specificity for common benign lesions like hemangiomas and simple cysts.

Conclusion: Ultrasound is a safe and effective method of detecting focal liver lesion. Its low cost, easy availability and lack of ionizing radiation and iodinated contrast media makes it most ideal for imaging the liver. It aids in defining therapeutic decision quickly and allows ultrasound guided interventions. High degree of specificity of ultrasound diagnosis in the present study confirms the value of ultrasonographic evaluation of focal liver lesions and suggests that it can be effectively used in the routine diagnostic work.
\end{abstract}

Keywords: Fine needle aspiration cytology, focal liver lesions, liver abscess, metastasis, ultrasound.

\section{Introduction}

Liver diseases are amongst the common causes of morbidity and mortality in India, which are encountered frequently in day-to-day practice. To establish the correct diagnosis and treatment, a precise initial diagnostic imaging modality is needed. Following history and clinical examination, ultrasonography has become one of the first and most useful methods of investigation in patients with upper abdominal pain, jaundice and mass per abdomen.

Ultrasound is widely accessible, inexpensive, non-invasive, and portable with high spatial and temporal resolution. Ultrasound is the first choice of investigation for screening of patients with suspected liver diseases. Focal liver lesions mainly comprise of liver abscess, cystic lesions, primary malignant neoplasms, metastases, focal fatty infiltrations and hematoma. The signs and symptoms of such lesions are non-specific and biochemical tests have limitations in the diagnosis of these lesions. Real-time ultrasonography has got considerable application in diagnosis of focal liver lesions. Ultrasonography has an important role in the detection and follow-up of focal liver lesions. It can be used as an imaging guide for FNAC and therapeutic drainage of abscesses.

\section{Aims Of The Study}

$>$ To evaluate the ultrasound imaging spectrum of focal hepatic lesions.

$>$ To study the relative prevalence of different focal hepatic lesions detected by ultrasound during the study period.

$>$ To evaluate the role of ultrasound in evaluation of focal hepatic lesions.

$>$ FNAC and/or CT scan correlation of ultrasound findings.

\section{Materials And Methods}

Place of study- Department of Radiology, P.D.U. Govt. Medical College and Civil Hospital, Rajkot, Gujarat, India.

Duration of Study- 3 months (1-09-2014 to 30-11-2014)

Type of Study- Prospective Study

Ultrasound Machines - Phillips IU22- Convex (1-5MHz), Linear (3-9MHz), Linear (5-17MHz)

and transducers used - Esaote MyLab 50 and Esaote MyLab 20- Convex (2.5-6.5MHz), and Linear (7.5$12 \mathrm{MHz}$ )

Inclusion criteria 
Cases of focal hepatic lesions detected by ultrasound during the study period.

Exclusion criteria

Diffuse fatty infiltration, Storage disorders, Cirrhosis of liver and Diffuse infiltrative malignancies, lymphoma and leukemia.

\section{Methodology}

Liver was scanned in various planes- sagittal, parasagittal, transverse, oblique, subcostal, intercostal and coronal planes. Comprehensive scanning of other upper abdominal organs was done. Various ultrasonographic features of focal liver lesions were observed, which include: Number of lesions - single or multiple, Location within liver - Lobar distribution (right lobe, left lobe, both lobes), segmental distribution, Echogenicity (by comparing with that of normal liver Parenchyma), hyperechoic, hypoechoic, anechoic or mixed echogenic. FNAC and/or CT scan correlation was done in all the patients.

\section{Results}

The study comprises of 50 cases of focal liver lesions studied by ultrasound. Patients with clinically suspected focal liver disease were referred to the Department of Radiology and incidental focal liver lesions were picked up in patients on whom ultrasonography was being done for other reasons. Findings were confirmed by FNAC or CT scan. The following observations were made.

Table 1- Distribution of cases diagnosed by ultrasound with FNAC/CT scan correlation

\begin{tabular}{|l|c|c|c|c|}
\hline \multicolumn{1}{|c|}{ Ultrasound diagnosis } & No. of Cases & Percentage & FNAC /CT scan & Specificity \\
\hline Liver abscess & 18 & $36 \%$ & 17 & $94.4 \%$ \\
\hline Metastases & 7 & $14 \%$ & 6 & $85.7 \%$ \\
\hline Hemangioma & 8 & $16 \%$ & 8 & $100 \%$ \\
\hline Cysts & 6 & $12 \%$ & 6 & $100 \%$ \\
\hline Hydatid lesion & 3 & $6 \%$ & 3 & $100 \%$ \\
\hline $\begin{array}{l}\text { Primary malignant liver } \\
\text { tumors }\end{array}$ & 4 & $8 \%$ & 3 & $75 \%$ \\
\hline Contusion/laceration & 4 & $8 \%$ & 4 & $100 \%$ \\
\hline TOTAL & $\mathbf{5 0}$ & $\mathbf{1 0 0 \%}$ & $\mathbf{4 7}$ & $\mathbf{9 3 . 6 \%}$ \\
\hline
\end{tabular}

Table 2- Age distribution of focal liver lesion

\begin{tabular}{|l|c|c|}
\hline Age group (years) & No. of Cases & Percentage \\
\hline Below 10 & 1 & 2 \\
\hline $11-20$ & 2 & 4 \\
\hline $21-30$ & 5 & 10 \\
\hline $31-40$ & 15 & 30 \\
\hline $41-50$ & 18 & 36 \\
\hline $51-60$ & 6 & 12 \\
\hline More than 60 & 3 & 6 \\
\hline Total & $\mathbf{5 0}$ & $\mathbf{1 0 0}$ \\
\hline
\end{tabular}

Table 3- Sex distribution of focal liver lesions

\begin{tabular}{|c|c|c|}
\hline Sex & No. of Patients & percentage \\
\hline Male & 31 & $62 \%$ \\
\hline Female & 19 & $38 \%$ \\
\hline Total & $\mathbf{5 0}$ & $\mathbf{1 0 0 \%}$ \\
\hline
\end{tabular}

Table 4- Distribution of cases based on number of focal lesions

\begin{tabular}{|c|c|c|}
\hline Lesions & No. of Patients & Percentage \\
\hline Solitary & 28 & $56 \%$ \\
\hline Multiple & 22 & $44 \%$ \\
\hline Total & $\mathbf{5 0}$ & $\mathbf{1 0 0 \%}$ \\
\hline
\end{tabular}

Table 5- Distribution of cases based on lobar involvement

\begin{tabular}{|c|c|c|}
\hline Lobar & No. of Patients & Percentage \\
\hline Right lobe & 28 & $56 \%$ \\
\hline Left lobe & 7 & $14 \%$ \\
\hline Both lobes & 15 & $30 \%$ \\
\hline Total & $\mathbf{5 0}$ & $\mathbf{1 0 0 \%}$ \\
\hline
\end{tabular}

\section{Discussion}

Ultrasonography has been an accepted method for the diagnosis of focal liver lesions because of its rapidity of diagnosis and its high sensitivity. Ultrasound features of focal liver lesions were studied and 
diagnosis was confirmed by fine needle aspiration cytology or CT scan. The various focal liver lesions encountered in the study were liver abscess, metastasis, primary malignant liver tumors, haemangiomas, cystic and hydatid lesions, Liver contusions and lacerations.

\section{Ultrasonographic Features Of Focal Liver Lesions}

Amoebic Liver Abscess: $80 \%$ of abscesses occur in right lobe due to streaming of portal venous blood from the more frequently and more heavily infected right side of colon and much greater volume of right lobe. Sonographically these are symmetrical, oval or rounded lesions with well-defined margins. They are usually peripherally situated touching the liver capsule (subcapsular location) ${ }^{[1]}$. Lesions are primarily hypoechoic compared to normal liver parenchyma, hence almost always shows distal acoustic enhancement, a feature that is less consistent with pyogenic abscess. Whenever an abscess is discovered by ultrasound, it should be followed by aspiration. Some lesions may show bizarre sonographic appearance like irregular echogenicity, interspersed with disorganized low level echoes. Ultrasonography can be used as a guide for aspiration in order to identify the causative organisms of liver abscess. Aspiration of contents from amoebic liver abscess shows characteristic anchovy sauce appearance ${ }^{[2]}$.

(Fig.1, Fig. 2, Fig. 3, Fig4)

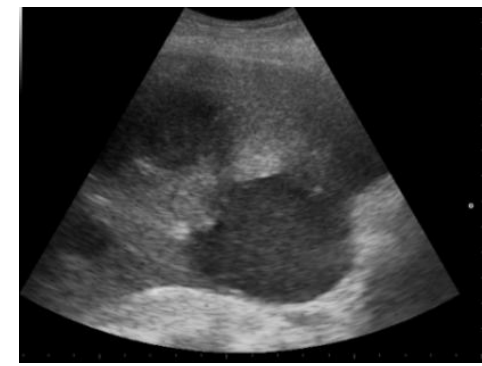

Fig.1- Non-liquefied multiple amoebic liver abscesses.

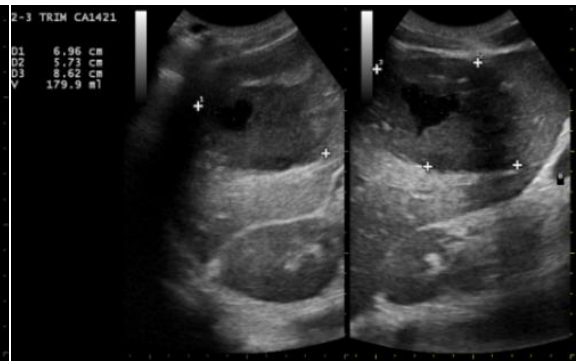

Fig.2- Partially liquefied amoebic liver abscess, right lobe.

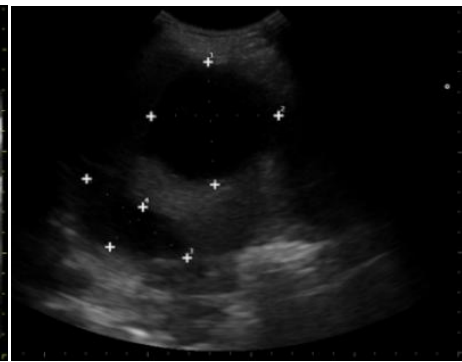

Fig.3- Completely liquefied abscesses.

Pyogenic Liver Abscess: Ultrasound will usually show spherical oval or slightly irregular echo poor lesions with distal enhancement. This pattern is present in $75 \%$ of cases. A significant number of abscesses can be higher in reflectivity than the adjacent normal liver. They are located usually at the center and rarely touching hepatic capsules. (Fig. 5)

Cysts: Ultrasonography is one of the most sensitive diagnostic modalities for the detection and characterization of cysts. Sonographically these cysts have thin well defined walls, are echo free and show distal acoustic enhancement. Morphologically they have smooth margins and essentially imperceptible walls and they lack septations ${ }^{[3]}$. The diagnosis of simple cyst was based on established ultrasonographic criteria like anechoic lesions, sharp smooth borders with strong posterior wall echoes, oval or spherical in shape and relative accentuation of echoes beyond the cyst compared to echoes at a similar depth. (Fig. 6)

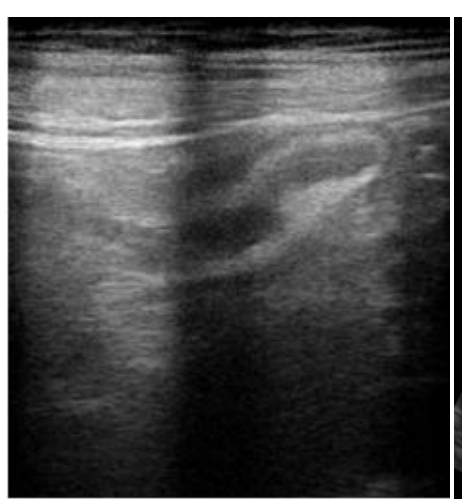

Fig.4 Associated Inflamed bowel loops at right iliac fossa

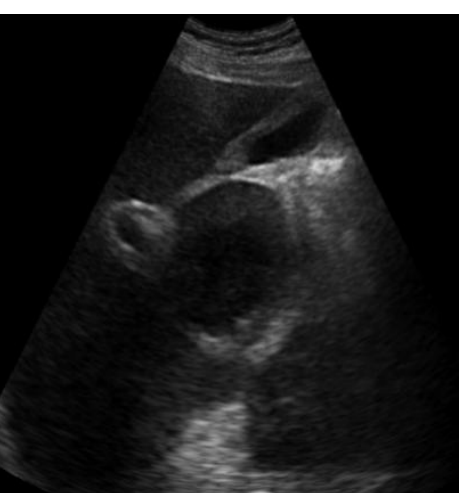

Fig.5- Pyogenic liver abscess.

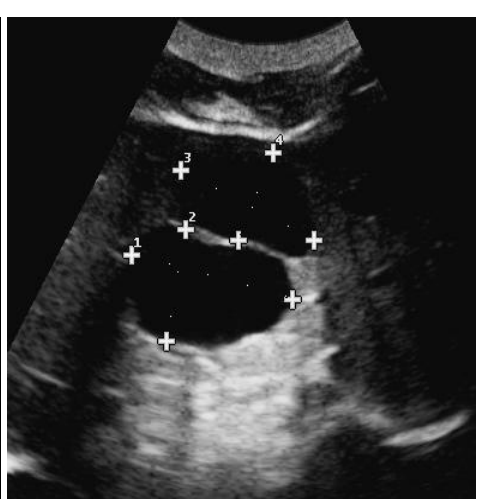

Fig. 6- Simple liver cysts 


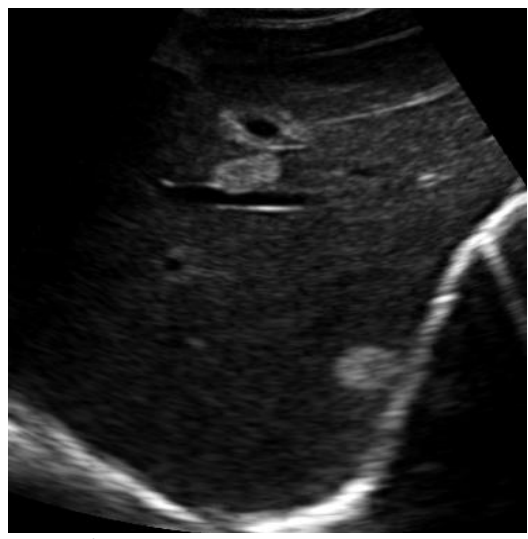

Fig. 7- Typical haemangiomas.

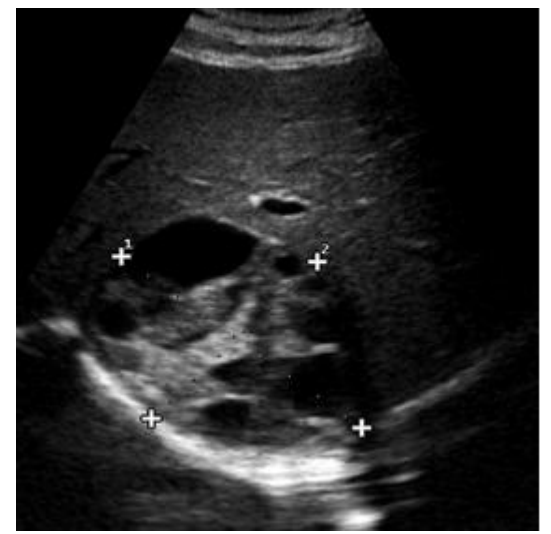

Fig. 8- Liver hydatid cyst showing multiple daughter cysts

\section{Hemangiomas}

Cavernous hemangioma is the most common primary liver tumour, occurring in $0.4-20 \%$ of the general population in autopsy series. ${ }^{[4]}$ Haemangiomas are most common in middle aged women (5:1), occur more frequently in the right lobe of the liver, are usually single and rarely greater than $5 \mathrm{~cm}$. At ultrasound, the most common appearance is of a well circumscribed, uniformly hyperechoic lesion. This is caused by multiple interfaces between walls of the cavernous spaces and blood within them. ${ }^{[5]}$ This classic appearance occurs in up to 70 per cent. In the remaining 30 per cent, definite diagnosis with ultrasound can be difficult and haemangiomas are a great mimic. (Fig. 7)

A minority of hemangioma may present as an isoechoic or hypoechoic mass relative to the liver parenchyma. Hemangiomas larger than $2.5 \mathrm{~cm}$ are reported to show posterior acoustic enhancement. Some hemangiomas, especially if they are large (more than $5-6 \mathrm{~cm}$ in diameter) present a heterogeneous ultrasound echo pattern. The anatomical basis for this is thought to be due to thrombosis, fibrosis, degeneration or hemorrhagic necrosis. Atypical appearance makes distinction from other focal lesions difficult.

\section{Hydatid Disease}

Liver is the most frequently involved organ with more than $50 \%$ of cysts found in the liver. Cysts are multiple in $40 \%$ of cases and about in $25 \%$ of patients with liver disease also have lung cysts. Lesions may be purely cystic, solid or mixed. The cyst appears as a well-defined sonolucent mass with smooth borders and good posterior enhancement. Wall calcification may occur years after the initial infection. The presence of complete rim of calcification suggests as inactive lesion. Debris, consisting of sand and scolices may be present within the hydatid lesion. Separation of the membrane producing an ultrasound "Water Lilly" sign results from detachment of inner germinal layer from the exocyst. This gives characteristic appearance for the hydatid lesion. The collapsed germinal layer is seen as an undulating linear collection of echoes either floating in the cyst or lying in the most dependent portion. The development of daughter cysts from the lining germinal layer produces a characteristic appearance of cyst within a cyst. This appearance is extensively characteristic, producing "cartwheel $\square$ or "honey comb" appearance. Another pattern is "Rossette" pattern, which is also characteristic appearance of a hydatid with daughter cysts. Capsule is well outlined but the inner architecture show circular array of cysts and a solid centre ${ }^{[6]}$. With heavy or continued infestation, multiple primary parent cysts may develop within the liver and will often produce hepatomegaly. Aspirated fluid from hydatid cyst is turbid and thick; fragments of the hyaline laminated cyst wall membrane are readily demonstrated. The diagnosis is confirmed by the demonstration of scolices or refractile hooklets ${ }^{[7]}$. (Fig. 8)

\section{Hepatocellular Carcinoma:}

$\mathrm{HCC}$ is the most common primary liver cancer comprising of $80 \%$ of primary liver malignancies. It is typically a disease of middle aged and elderly individuals. The sonographic appearance of $\mathrm{HCC}$ is variable. The masses may be hypoechoic, complex or echogenic. Most small $(<5 \mathrm{~cm}) \mathrm{HCC}$ are hypoechoic. A thin peripheral hypoechoic halo, which corresponds to a fibrous capsule, is seen most often in small HCC. With time and increasing size, the masses tend to become more complex and heterogeneous as a result of necrosis and fibrosis. Calcification is uncommon. Small tumors may appear diffusely hyperechoic, secondary to fatty metamorphosis or sinusoidal dilatation, making them indistinguishable from hemangioma. Ultrasonography provides information on shape, echogenicity, growth pattern and vascular involvement of the neoplasm. Three different shapes may be identified, depending upon the size and the invasiveness of the neoplasms: nodular, massive and diffuse. The echogenicity is variable and the tumor mass may appear hypo, hyper or isoechoic in comparison 
with the surrounding liver tissue. A mixed pattern and/or a hypoehcoic ring may also be visualized. Finally, ultrasound guidance allows puncture of intrahepatic nodules as small as $1 \mathrm{~cm}$. The sensitivity of this procedure in the diagnosis of focal liver lesions is very high, varying between $91 \%$ and $95 \%$ with a specificity of $92 \%-100 \%$ [7]. (Fig. 9, Fig. 10)

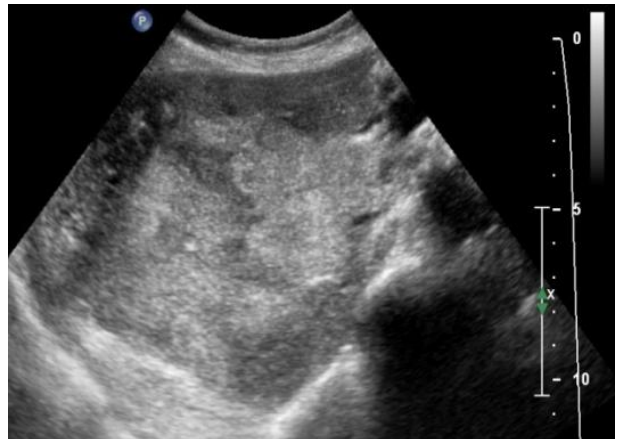

Fig. 9-Hepatocellular Carcinoma. Large heterogenous mass

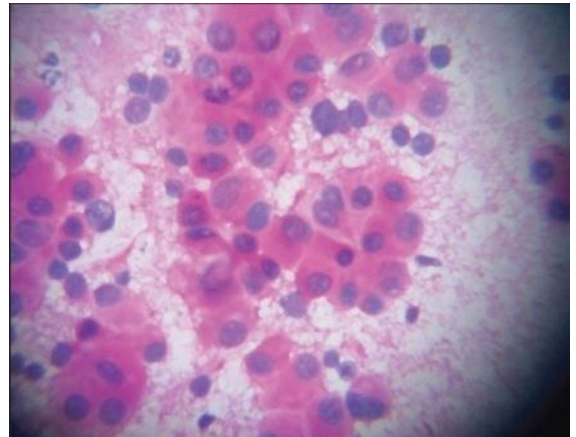

Fig.10-FNA smear of same patient showing features of Hepatocellular carcinoma.

\section{Metastasis}

The liver is one of the commonest sites for metastasis and terminal involvement is the rule in all but CNS malignancies. This can be attributed to its large size, high rate of blood flow and double perfusion by the portal vein and hepatic artery. The route of tumor spread to the liver is more likely to be hematogenous rather than lymphatic, because for the most part of the liver's lymphatics are hepatofugal. The most common primary tumors are those of the gut, breast, lung and melanoma. Metastasis may be found in any part of the liver and are usually multiple. The wide ranges of appearances are encountered in liver metastatic disease. Focal lesions are commonest but the malignancy may also infiltrate widely. The commonest focal pattern is of echopoor masses. Highly reflective lesions may be surrounded by an echo-poor band which may be fine or a few millimeters thick. This is called Bull's eye pattern and is more often seen in larger lesions. Highly reflective and target lesions are typically of tumors originating in the gastrointestinal tract and urogenital tract. These are recognized by distal enhancement caused by them. They may contain clear fluid as may be produced by mucin secreting lesion (e.g., cancer pancreas/ ovary) but contain debris when the fluid represents tumor necrosis. These types tend to have shaggy walls and are less likely to be mistaken for simple cysts ${ }^{[8]}$. Calcified lesions have very intense echoes and may show shadowing if the foci are sufficiently large. A difficult differential diagnosis is that from hemangiomas which also have a variety of echo patterns. Definite differential diagnosis is not possible based on ultrasound alone. (Fig. 11, Fig. 12, Fig. 13)

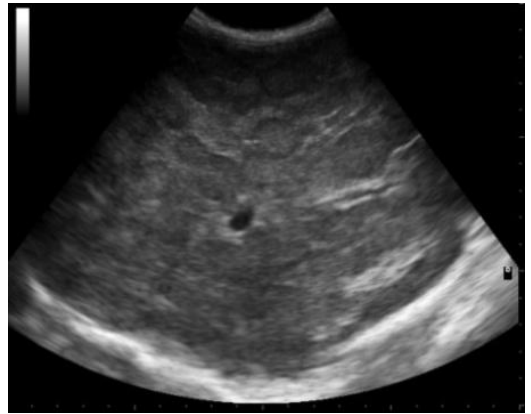

Fig.10- Multiple metastasis from from Ca breast. Multiple hypoechoic and target like lesions.

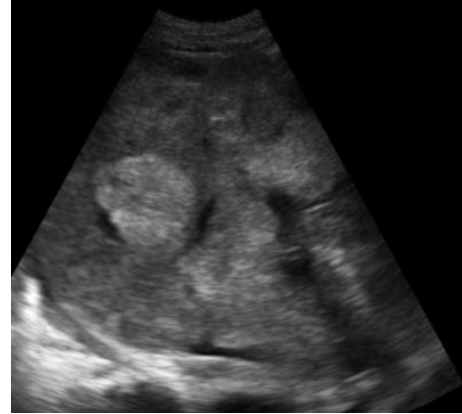

Fig.11- Multiple metastasis from Fig.12- FNA smear from liver nodule renal cell carcinoma.

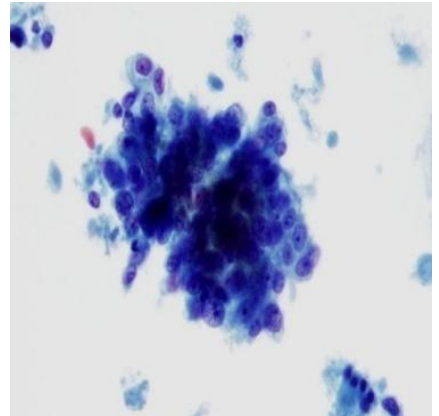

from patient with Ca breast showing adenosquamous differentiation.

\section{Conclusion}

Ultrasound is a safe and effective method of detecting focal liver lesion. Its flexibility, easy availability and lack of dependence on organ function makes it most ideal for imaging the liver and also serves as an object of defining therapeutic decision quickly. Ultrasonography when adopted as an initial imaging modality was seen as a method which reduced the cost and time to arrive at a diagnosis. By this rapid method, even small lesions with subtle difference in reflectivity can be detected. The liver can be scanned in multiple planes enabling us to know the exact location of lesions and study their echo pattern. Apart from detecting lesion, other valuable information like ascites, vessel involvement, primary source of malignancy in abdomen and pelvis can be easily 
obtained. Ultrasonography is highly sensitive in diagnosing focal liver lesions such as Liver abscess, metastases and primary malignant liver tumors and hydatid lesions which constituted majority of focal liver lesions for which ultrasound was indicated in the present study, with a specificity of $94.4 \% \%, 85.7 \% \%, 75 \%$ and $100 \% \%$ respectively. The great majority of asymptomatic, single lesions are benign. Hepatic cysts and haemangiomas with a classic appearance can be safely diagnosed with ultrasound alone.

It is evident from this study that ultrasonography has a wide applicability in the diagnosis of focal liver lesion. Being a safe, simple, repeatable and without radiation exposure to the patient, it is worthy of being included in routine diagnostic work. In spite of the advent of newer diagnostic modalities, it still holds a unique status even in the current perspective. Validity of ultrasonographic diagnosis in relation to FNAC diagnosis and/or CT scan was done in 50 cases of focal liver lesions. There is a significant association between USG findings and FNAC diagnosis. High degree of sensitivity and specificity of USG diagnosis in the present study confirms the value of ultrasonographic evaluation of focal liver lesions and suggests that it can be effectively used in the routine diagnostic work.

\section{References}

[1]. Kuligowska E, Noble J. Sonographic features of hepatic abscess. Semin Ultrasound. 1983;4: 102-116

[2]. Gossin KB. Intrahepatic focal liver lesions -Differential diagnosis. Am J Roentgenol.1981; 137: 763-767.

[3]. Edmonston HA, Peters RL. Tumors of the liver - Pathological features. Seminars in Roentgenology. 1983; 18(2): 75-83.

[4]. Gaines P A, Sampson M A. The prevalence and characterisation of simple hepatic cysts by ultrasound examination. Br.J.Radiol. 1989;62:335-7.

[5]. Liang P, Cao B, Wang Y, Yu X, Yu D, Dong B. Differential diagnosis of hepatic cystic lesions with grey-scale and colour Doppler sonography. JClin Ultrasound. 2005;33(3):100-5.

[6]. TareqSinan, Mehraj Sheik, Abdulla Behbdoni, Fayaz A Chisti, Zafar Sheik, PR Hira et al. Diagnosis of abdominal hydatid cyst: The role of ultrasound and ultrasound guided fine needle aspiration cytology. International Journal of Medical Principles and Practice. 2002; 11(04).

[7]. Bolandi L, Gaiani S, Benzi G, Zironi G, RigamontiA,Fuscorni F et al. Ultrasonography and guided biopsy in the diagnosis of hepatocellur carcinoma. Italian J of Gastroenterology. Jan. 1992; 24(1): 46-49.

[8]. Scheible W, Gosink BB, Leopold GR. Gray scale echographic patterns of hepatic metastatic disease. Am J Roentgenol. 1977; 129: 983-987 